\title{
Higgs Boson Physics at CMS
}

\author{
Predrag Cirkovic ${ }^{1, \star}$ (on behalf of the CMS Collaboration) \\ ${ }^{1}$ Institute of Physics Belgrade, Pregrevica 118, P.O. Box 68, 11080 Belgrade, Serbia
}

\begin{abstract}
An overview of the standard model Higgs boson analyses and beyond standard model Higgs boson searches will be given. A data sample of proton-proton collisions at $\sqrt{s}=13 \mathrm{TeV}$ collected with the CMS detector at the LHC is used. The measurements of properties of the Higgs boson in the $4 \ell$ and $\gamma \gamma$ decay channels will be presented. The future prospects will be covered.
\end{abstract}

\section{Introduction}

In this report, the subjects will be discussed in the three main sections. After a brief experimental and theoretical overview, a presentation of the latest results of the Standard Model (SM) Higgs boson measurements in the main analysis channels together with the measurements of the Higgs boson properties thereafter, will be discussed in section 2. The latest results of the searches for beyond the Standard Model (BSM) Higgs boson in terms of the charged, MSSM, doubly-charged, invisible and di-Higgs boson production, will be covered in section 3. The future prospects for the SM Higgs boson production in the $4 \ell$ and $\gamma \gamma$ decay channels will be described in section 4 . The summary will be described at the end. The focus will be on the most recent results, such as the Higgs boson mass measurement in the $H \rightarrow 4 \ell$, fiducial cross section measurements in the $4 \ell$ and di-photon channels, the latest results on $t \bar{t} H$, and the search for resonant and non-resonant di-Higgs boson production. Many of the searches at CMS [1] presented here have been updated with the full 2016 dataset corresponding to an integrated luminosity of $35.9 \mathrm{fb}^{-1}$.

\section{Standard Model (SM) Higgs Boson Searches}

Starting with the measurements of the Higgs boson production and decay rates with the combined 7 and $8 \mathrm{TeV}$ CMS dataset [2] and the combined ATLAS and CMS analysis of the LHC pp collision data at $\sqrt{s}=7$ and $8 \mathrm{TeV}$ [3], the measured signal strenghts, both the individual ones measured by CMS and ATLAS alone and the combined one are compatible with 1.0. The largest uncertainties for the production modes appear in the channel with the lowest cross sections, such as $t \bar{t} H$. Concerning decays, the smallest uncertainties are present in the channels with the cleanest signatures, e.g. $H \rightarrow \gamma \gamma$ and $H \rightarrow W W$, and the largest uncertainties in $H \rightarrow b \bar{b}$ are caused mostly by the presence of large QCD background. Individual signal strengths $(\mu)$ have been also measured in the plane of their fermionic and bosonic parts, with the combined CMS [2] and the combined CMS and ATLAS [3] data from the

\footnotetext{
^e-mail: predrag.cirkovic@cern.ch
} 
LHC pp collision at $\sqrt{s}=7$ and $8 \mathrm{TeV}$. An overall agreement of the 2D maximum likelihood best fits with the SM expectations has been achieved for all channels within $1 \sigma$ region.

The same datasets used in Refs. [2] and [3] have been used to measure also the coupling between the Higgs boson and the other SM particles. The measurements of the coupling with muon, $\tau$-lepton, b-quark, $W$ and $Z$ bosons and top quark have been compared with their prediction, which are proportional to the particle mass. The coupling values range between very small values, featuring muons, up to the values close to unity, which characterize the top quark. An overall agreement between the measurements and the theoretical prediction has been noticed here as well. The Run I measurements already put constraints on the couplings and for the measurements with the Run II datasets it is expected to get more stringent constraints. The fits in the $2 \mathrm{D}$ plane of the coupling modifiers for vector bosons $\left(\kappa_{V}\right)$ and fermions $\left(\kappa_{F}\right)$ show that the results are compatible with their SM expectations $(1,1)$ for all five Higgs boson decay channels and their combination within one standard deviation $(1 \sigma)$.

\subsection{Higgs boson decaying to a pair of photons}

$H \rightarrow \gamma \gamma$ [4] represents one of the Higgs boson discovery channels, characterized by high resolution with a very small uncertainty on di-photon mass, a clean final state, and a small branching ratio. Boosted decision tree is used for the photon identification, selection of di-photon vertices, and selection of di-photon events. Boosted Decision Tree (BDT) distribution has been flattened according to the total $\gamma \gamma$ signal [4], which is composed of the four Higgs boson production mode components. In this report, the Fig. 5 (left) of [4] has been presented. The distribution of the transformed score of the di-photon multivariate (BDT) classifier for events with two photons satisfying the preselection requirements in data has been compared to the distribution of the simulated signal and simulated background stacked together [4]. The correspondence between the data distribution and the distribution of the total signal plus background stack has been achieved. The score of the BDT classifier is used to select and divide the events into four "untagged" categories according to the di-photon mass resolution and predicted signal over background ratio. The category of the events with the lowest BDT score has been discarded from the analysis. The signal is selected according to the highest transverse energy photons and the background is composed of the irreducible $\gamma \gamma$ component, and the reducible fakes that originate from $\gamma+$ jet and di-jet events. In Fig. 1 (left), a di-photon mass distribution is presented, for the total signal and background (upper row in Fig. 1, left) and for the background component subtracted (bottom row in Fig. 1, left). The best fit mass is found at $m_{H}=125.4 \mathrm{GeV}$ with statistical uncertainty of approximately $0.15 \mathrm{GeV}$ and the systematic uncertainties preliminarily estimated to be between $0.2 \mathrm{GeV}$ and $0.3 \mathrm{GeV}$ (still under study) [4]. In Fig. 1 (right), the results of the signal strength measurements in the $H \rightarrow \gamma \gamma$ are presented. The signal strength measurement for the four categories combined provide the value of $1.16_{-0.14}^{+0.15}$, compatible with the SM prediction within $\approx 1 \sigma$. The gluon fusion signal strength is the most compatible one, while the $t \bar{t} H$ providing the signal strength of 2.2, corresponds to a $3.3 \sigma$ excess with respect to the absence of $t \bar{t} H$ production, and agrees with the SM prediction within $1.6 \sigma$.

The signal strength measured in bosonic and fermionic parts [4] is also consistent with the SM predictions, especially in its fermionic part, which is mostly driven by contribution from gluon fusion process. The 2D likelihood scans of the Higgs boson coupling modifiers have been also performed in $H \rightarrow \gamma \gamma$, but here for the couplings to photons and gluons, and couplings to vector bosons and fermions [4]. Both fits are compatible with the SM predictions, within the range of $1 \sigma$.

The Fig. 2 summarizes the most recent results on the fiducial cross section measurements performed in $H \rightarrow \gamma \gamma$ channel [5]: the differential fiducial cross section measurements for di-photon $p_{T}$, in Fig. 2 (left), and the number of additional hadronic jets, in Fig. 2 (right) [5]. For this measurement, CMS requires two high- $p_{T}$ isolated photons within the fiducial volume defined as: $p_{T 1} / m_{\gamma \gamma}<1 / 3$, 

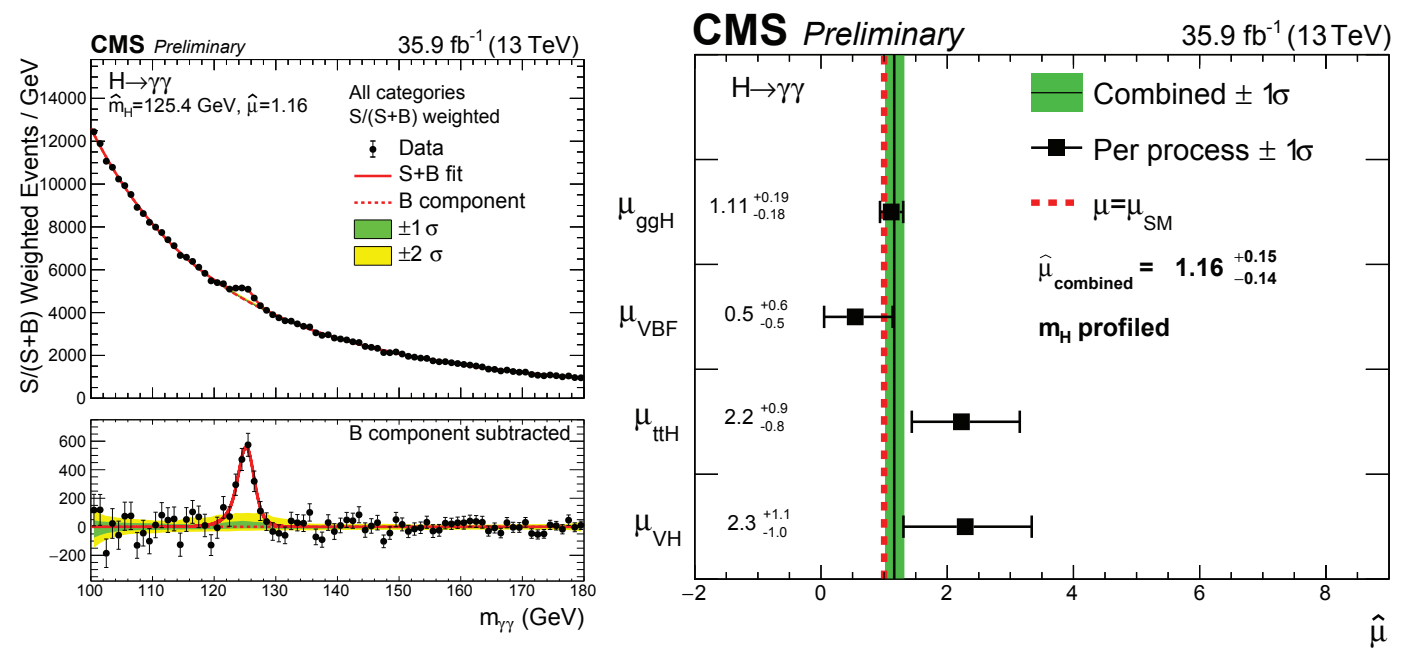

Figure 1. The $m_{y \gamma}$ distribution (left) as weighted sum of all categories [4]. S and B represent the number of signal and background events, respectively. Signal strength modifiers (right) measured for each process (black points) for profiled $m_{H}$, compared to the overall signal strength (green band) and to the SM expectation (dashed red line) [4].
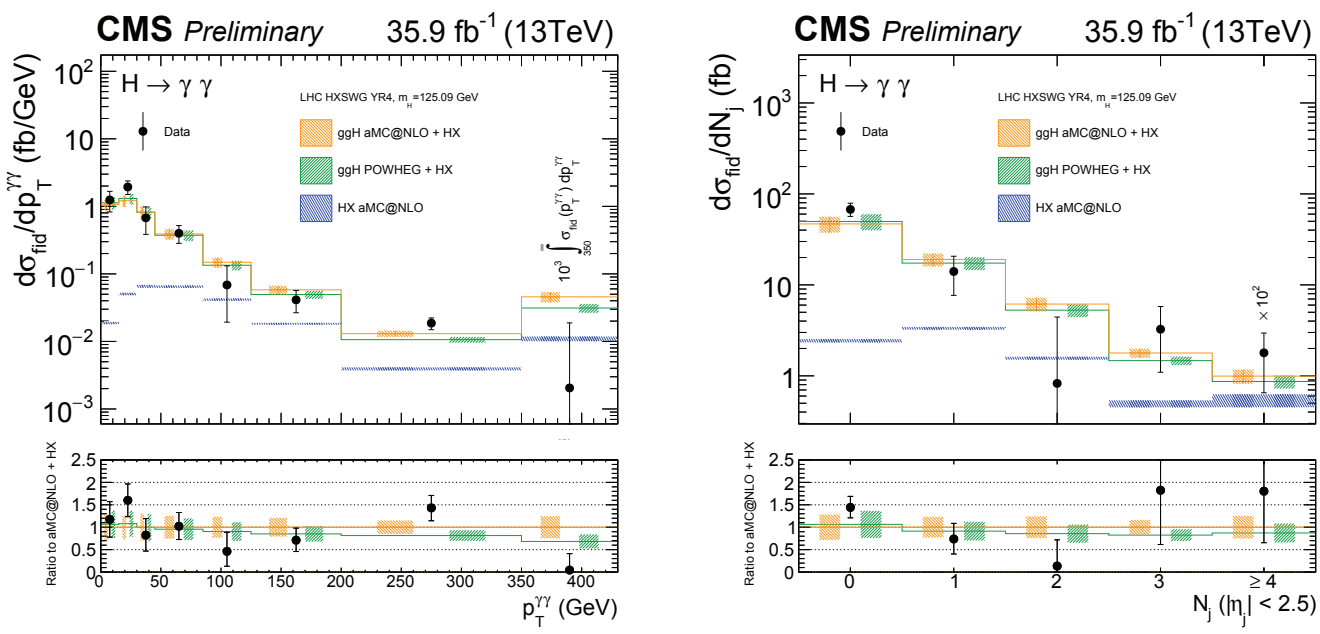

Figure 2. Measured $H \rightarrow \gamma \gamma$ differential cross-section (black points) for $p_{T}{ }^{\gamma \gamma}$ (left) and $N_{\text {jets }}$ (right) [5]. The measurements are compared to the theoretical predictions, combining the Higgs boson cross sections and branching fraction as in the LHC Higgs Cross Section Working Group [6].

$p_{T 2} / m_{\gamma \gamma}<1 / 4,\left|\eta_{1,2}\right|<2.5$ I $_{\text {so }} o_{g e n 1,2}<10 \mathrm{GeV}(\Delta R=0.3)$. No classification has been performed according to production mode, which implies the focus on gluon fusion. Classification of events based on mass resolution estimator improved sensitivity by $10 \%$. The signal production cross section is extracted through simultaneous maximum likelihood fit to the di-photon invariant mass in all the 
production modes. The unfolding to particle-level cross sections is achieved by extracting the vector of fiducial cross sections directly from the likelihood fit. The result of the total fiducial cross section with the $13 \mathrm{TeV}$ data, measured to be $\hat{\sigma}_{\text {fiducial }}=84 \pm 11$ (stat) \pm 7 (syst) $=84_{-12}^{+13}$ [5] (and compared to the result obtained with the $8 \mathrm{TeV}$ data), shows no significant deviations from the SM predictions and represents the most precise fiducial measurement to date.

\subsection{Higgs boson decaying to four leptons}

The next important discovery channel is $H \rightarrow Z Z \rightarrow 4 \ell$ [7], with the signature consisting of four isolated leptons (electrons or muons), with excellent momentum resolution. The leptons are grouped into pairs where each of them contains the leptons of the same flavour and the mutually opposite sign of the charge. This channel is characterized by a fully reconstructed $4 \ell$ mass peak, shown in Fig. 3 (left), and a large signal over background ratio $(S / B>2: 1)$. The main background, estimated from Monte Carlo, is irreducible SM production of $Z Z$, and the reducible background, estimated from data, is $Z+X$, which is dominated by fakes from $Z+$ jets. A kinematic discriminants $\left(D^{k i n}\right)$ based on matrix element calculations is used for the signal extraction. The events are split into seven categories according to Higgs boson production modes and based on the number of leptons, number of (b-)jets, transverse missing energy and the selection on the kinematic discriminant. Signal strength in this channel is measured for each of the categories and each of the production modes providing consistent combined results [7], as well as in the plane of its fermionic and bosonic parts, in Fig. 3 (right). All the results show agreement between the measurements and the SM predictions within the present uncertainties.
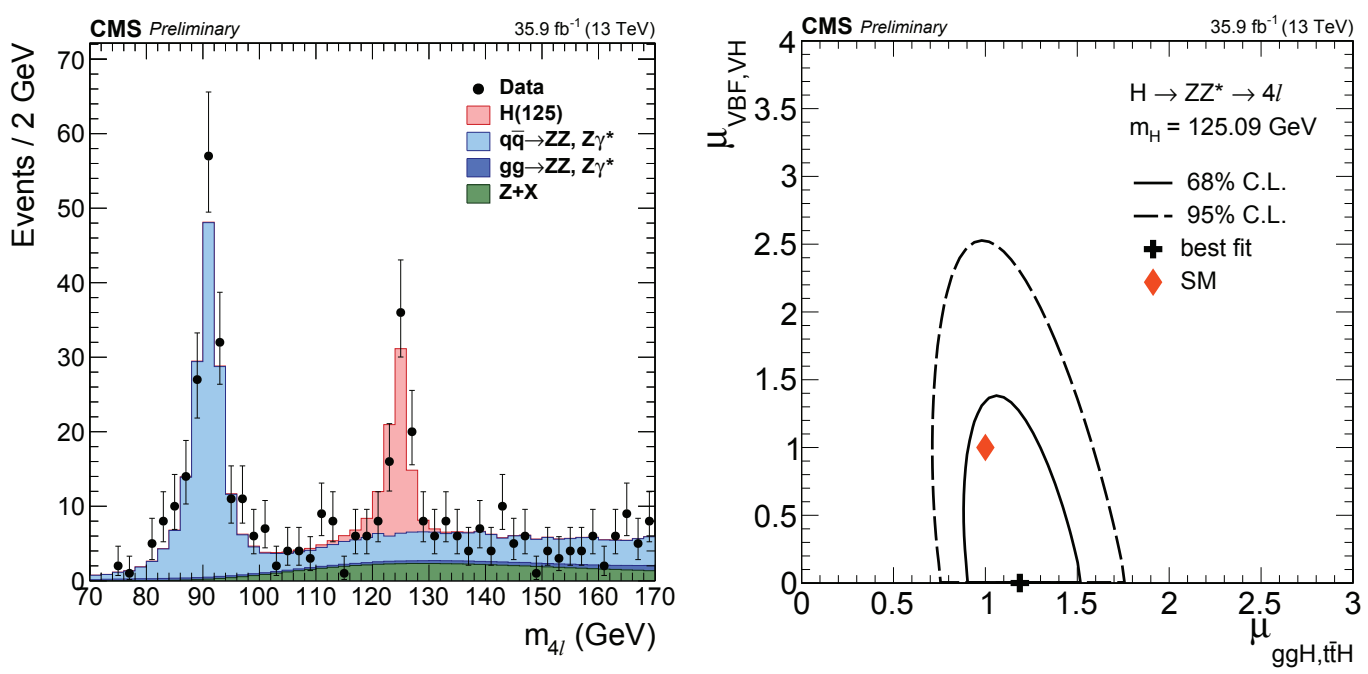

Figure 3. Distribution of the reconstructed four-lepton invariant mass $m_{4 \ell}$ in the low-mass range (left) [7]. Points with error bars represent the data and stacked histograms represent expected signal and background distributions. Result of the 2D likelihood scan for the $\mu_{g g F, t \bar{H} H}$ and $\mu_{V B F, V H}$ signal strength modifiers (right) [7], where $g g F$ (or $g g H$ ) denotes gluon fusion production mode, $t \bar{t} H$ denotes the production of the Higgs boson associated top quarks, VBF denotes vector boson fusion production, and VH denotes production of the Higgs boson associated to a (weak) vector boson. The solid and dashed contours show the $68 \%$ and $95 \%$ confidence level (CL) regions, respectively. 

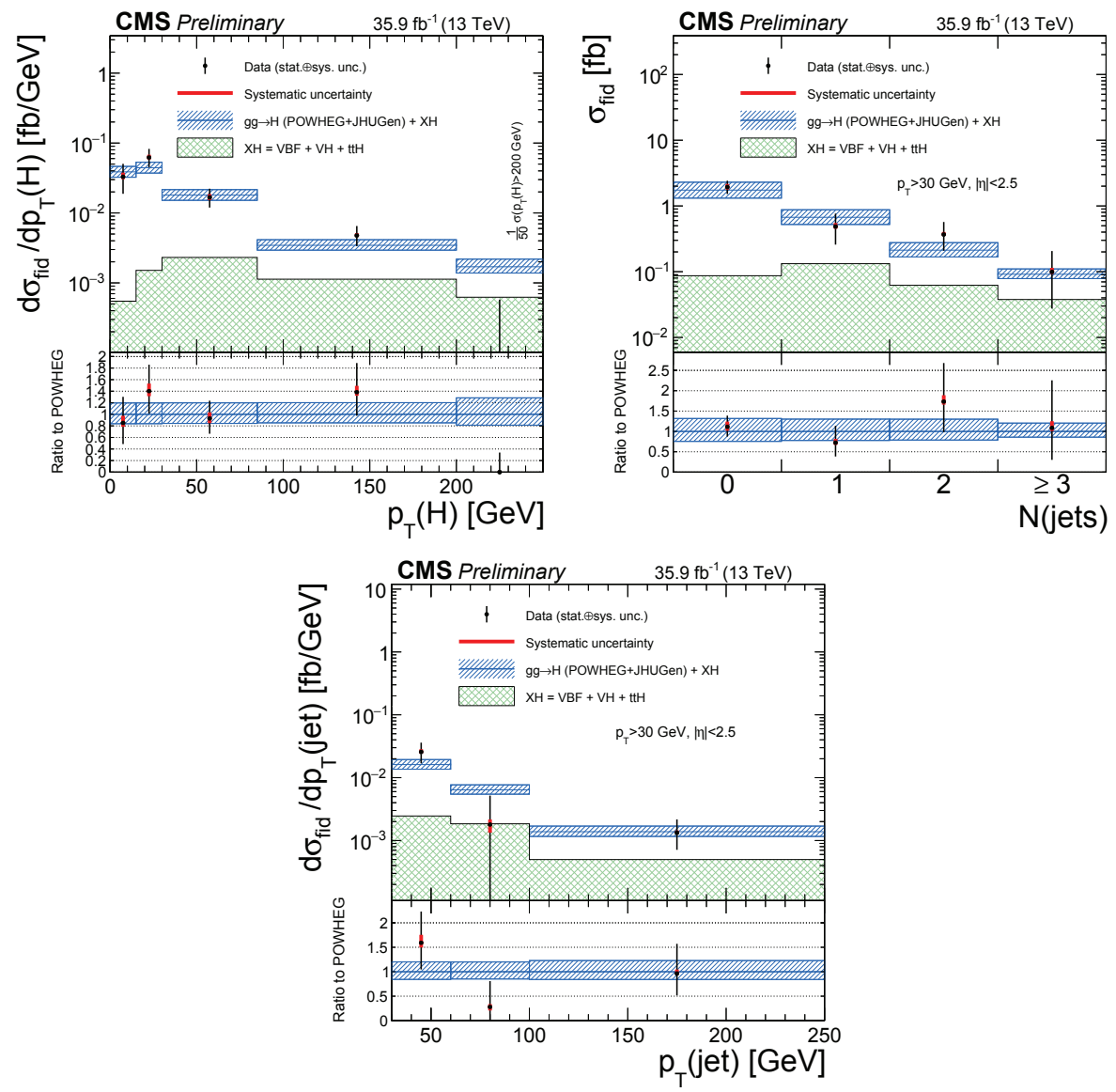

Figure 4. The results of the differential cross section measurement for $p_{T}(H)$ (top-left), $N$ (jets) (top-right) and $p_{T}($ jet $)$ (bottom). The acceptance and theoretical uncertainties in the differential bins are calculated using POWHEG. The sub-dominant component of the signal $(V B F+V H+t \bar{t} H)$ is denoted as $X H$ [7].

Fiducial cross section measurement has been performed in the $H \rightarrow Z Z \rightarrow 4 \ell$ channel. This measurement is characterized by minimal dependence on the assumptions of the relative fraction or kinematic distributions of the separate production modes. The details about the fiducial volume definition in form of the lepton kinematics and isolation requirements and the event topology requirements, are summarized in the Tab. 4 of [7]. This definition is very similar to the one used for the fiducial cross section measurement performed at 7 and $8 \mathrm{TeV}$ [8], however the new measurement provides better agreement with the SM expectations and lower (statistics-dominated) uncertainties. The differential cross sections has been measured in $H \rightarrow Z Z \rightarrow 4 \ell$ versus $p_{T}$ of the Higgs boson, jet multiplicity and $p_{T}$ of the jet. The dominant sources of systematic uncertainty are the experimental uncertainties of the lepton identification efficiencies and luminosity measurement, and the theoretical uncertainties are found to be sub-dominant. In order to asses the model dependence of the measurement, the unfolding procedure is repeated using different response matrices created by varying the relative fraction of each SM production mode within its experimental constraints. The uncertainty is determined to be negligible with respect to the experimental systematic uncertainties. The results of the differential 
cross section measurement in Fig. 4 show a relatively good agreement between data and gluon fusion dominated predictions from POWHEG [9-11] simulation.

\subsection{Higgs boson mass and width measurements}

Mass measurement performed in $4 \ell$ channel [7] is based on one-, two- and three-dimensional fit on the following variables: invariant mass of the Higgs boson $\left(m_{4 \ell}\right)$, expected uncertainty on mass $\left(\mathcal{D}_{\text {mass }}\right)$ and the kinematic discriminant $\left(\mathcal{D}_{b k q}^{k i n}\right)$. In Fig. 5 (left), there is a comparison of the 1D likelihood scan as a function of mass for the 1D, 2D, and 3D measurement. The likelihood to be maximized is constructed using the reconstructed transverse momenta of the two leptons forming the $\mathrm{Z}$ candidate with the invariant mass closest to the nominal $\mathrm{Z}$ boson mass (Z1), the per lepton resolutions, the refitted transverse momentum and the invariant mass calculated from the refitted four momenta. For each event, the likelihood is maximized and the refitted transverse momenta are used to recalculate the four-lepton mass and mass uncertainty, which are denoted as $m_{4 l}^{\prime}$ and $D_{\text {mass }}^{\prime}$. These distributions are then used in the definition of the likelihood to extract the Higgs boson mass [7]. Compared to the mass measurements performed with the CMS and ATLAS Run I data [12], which combination was already quite precise (less than $0.2 \%$ ), the new result is even more precise (around $0.17 \%$ ). The latest measurement presented in Fig. 5 (right), performed in the mass range between 105 and $140 \mathrm{GeV}$, using the on-shell Higgs boson production and the floating $m_{H}$ parameter, constrained the Higgs boson width to be less than $1.1 \mathrm{GeV}$ at $95 \%$ C.L [7].
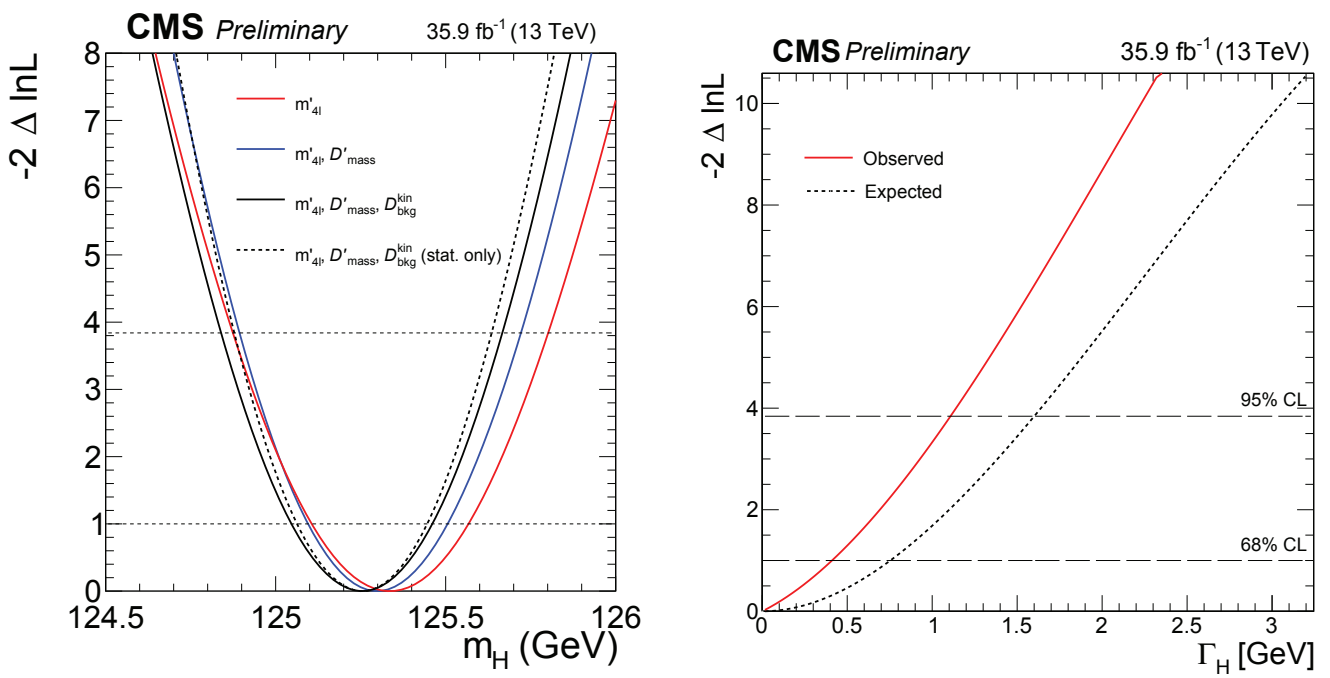

Figure 5. 1D likelihood scan as a function of mass for the 1D, 2D, and 3D measurement (left). The likelihood scans are shown for the mass measurement using the refitted mass distribution with $m(Z 1)$ constraint. Solid lines represents the scan with full uncertainties included, dashed lines statistical uncertainty only [7]. Observed and expected likelihood scan of $\Gamma_{H}$ using the signal range $105<m_{4 \ell}<140 \mathrm{GeV}$, with $m_{H}$ floated [7].

\subsection{Higgs boson decaying to a pair of tau leptons}

Higgs boson decaying to $\tau \tau$ [13] is another discovery channel, because of the large event rate expected in the SM with respect to the other leptonic decay modes, and the smaller contribution from 
background events with respect to the $H \rightarrow b \bar{b}$ channel. With the four final states $\left(\mu \tau_{h}, e \tau_{h}, \tau_{h} \tau_{h}\right.$, and $e \mu$ ), split into three categories ( 0 -jet, VBF and boosted), the total number of categories reaches twelve. In each category the two variables are chosen to build two-dimensional (2D) distributions. The search for an excess of the signal events over the expected background involves a $2 \mathrm{D}$ global maximum likelihood fit based in all channels, together with control regions for the $t \bar{t}$, QCD multijet, and $\mathrm{W}+\mathrm{jets}$ backgrounds. Combined observed and predicted $m_{\tau \tau}$ distributions that include the VBF category of the $e \mu, e \tau_{h}$ and $\mu \tau_{h}$ channels are shown in Fig. 6 (left). The signal is normalized to its best-fit signal strength, while the normalization of the predicted background distributions $(Z \rightarrow \tau \tau, \mathrm{W}+\mathrm{jets}, \mathrm{QCD}$ multijet and others) corresponds to the result of the global fit. The mass distributions for a constant range of the second dimension of the signal distributions are weighted according to $S /(S+B)$. The "others" background contribution includes events from diboson, $t \bar{t}$, and single-top quark production, as well as scalar boson decays to a pair of $\mathrm{W}$ bosons and $\mathrm{Z}$ bosons decaying to a pair of light leptons. The background uncertainty band includes all systematic and statistical sources of background uncertainties, after the global fit. The inset in the top-right corner in Fig. 6 (left) shows the corresponding difference between the observed data and the sum of the expected signal and background distributions.

The final results are presented in the form of the plots of the signal strength per category and the signal strength per channel, with the combined best-fit on the signal strength of $1.06 \pm 0.25$ [13]. A graph of the p-value versus mass of the Higgs boson, in Fig. 6 (right), presents the observation of decays of the SM scalar boson to pairs of $\tau$ leptons, with an observed significance of 4.9 and an expected significance of 4.7 standard deviations for a mass of $125 \mathrm{GeV}$.
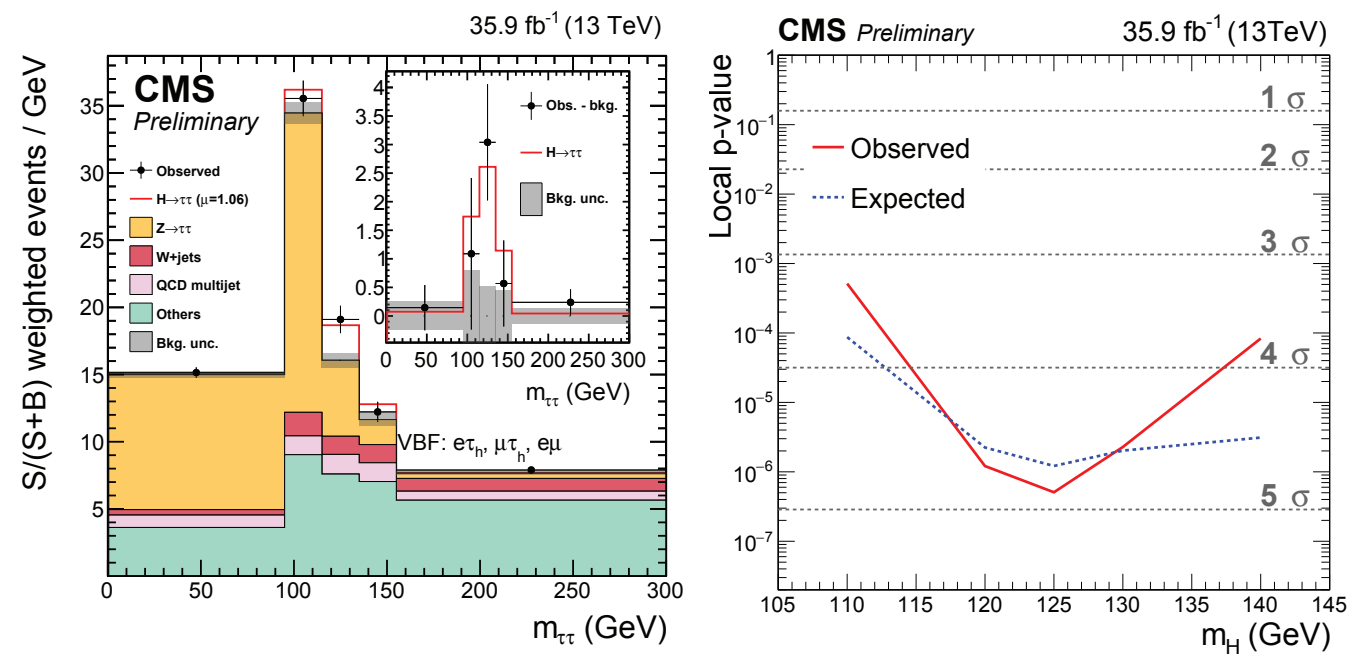

Figure 6. Combined observed and predicted $m_{\tau \tau}$ distributions, together with the inset showing the difference between the observed data and the sum of the expected signal and background distributions (left) [13]. Local p-value and significance as a function of the SM scalar boson mass hypothesis (right). The observation (red) is compared to the expectation (blue) for a scalar boson with a mass $m_{H}=125 \mathrm{GeV}$ [13].

\subsection{Production of the Higgs boson associated to top quarks}

The latest results of the analyses of the production of the Higgs boson associated to a pair of top quarks have been also presented. Concerning its large number of possible final states, the several channels 


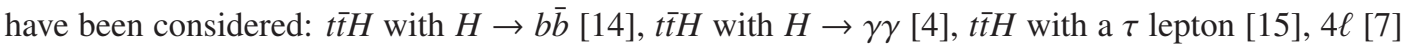
and multilepton [16] in the final state. All except the $t \bar{t} H$ with $H \rightarrow b \bar{b}$ have been updated with the full 2016 dataset. In $H \rightarrow \gamma \gamma$, and the $t \bar{t} H$ to multilepton, the significance exceeds $3 \sigma$. The plot in Fig. 7 summarizes the results of the signal strength measurements performed with Run II data from all channels. The comparison with the combined result of the analyses obtained with the Run I CMS and ATLAS data is presented [3].

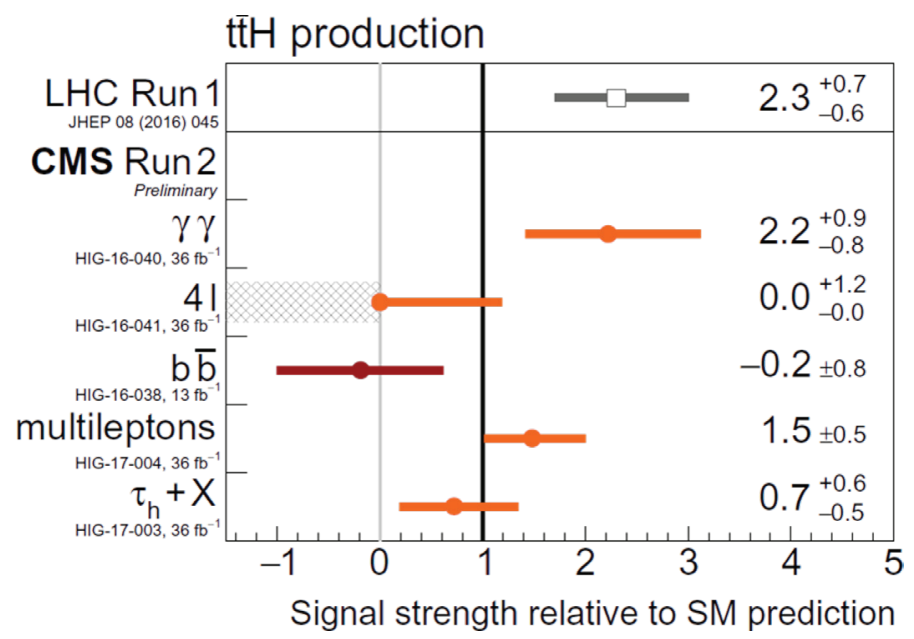

Figure 7. The $t \bar{t} H$ analyses summary plot for the signal strengths relative to the SM prediction.

The $t \bar{t} H$ analysis with $H \rightarrow b \bar{b}$ [14] considers the two following final state categories: di-lepton and lepton plus jets. Dilepton channel is characterized by minimal non-t $\bar{t}$ background and minimal jet combinatorics, while the lepton plus jets is characterized by high statistics. In Fig. 8 the results of the signals strength measurements and the $95 \%$ CL upper limits on $\mu$ are shown.

The $t \bar{t} H$ to multilepton [16] is performed with the Higgs boson decays to $W W, Z Z$ and $\tau$ leptons characterized into the three final state categories: two leptons same sign $(2 \ell s s)$, three leptons $(3 \ell)$ and four leptons $(4 \ell)$. Signal is extracted using the unrolled 2D BDT distributions, where the BDT discriminators have been trained separately against $t \bar{t}$ and $t \bar{t} V$. This channel has provided an evidence for the $t \bar{t} H$ production, giving the result on the signal strength of about $1.5+/-0.5$, which could be noticed from the excess of events in the di-lepton channel, in Fig. 9 (left), spread across the bins.

Higgs boson decaying to at least one $\tau$ [15] lepton in the final state is one of the $t \bar{t} H$ channels complementary to the $t \bar{t} H$ to multilepton channels. It considers the $1 \ell+2 \tau_{h}, 2 \ell s s+1 \tau_{h}$ and $3 \ell+1 \tau_{h}$ event categories, with the $t \bar{t} Z / W$ and electroweak background, as well as the fakes coming from the non-prompt leptons, jets to $\tau_{h}$ and the charge-flips as the main background components. The results have been observed with the significance of $1.4 \sigma$, while the expected significance is $1.8 \sigma$. The upper limit on the signal rate of 2.0 times the SM $t \bar{t} H$ production rate at $95 \%$ CL is set [15].

The search for the Higgs boson is also performed in the channel with a single top quark associated to the Higgs boson. This production is characterized by a relatively low cross section with respect to the total $t \bar{t} H$ cross section. In SM, this is the consequence of a destructive interference of the production with the coupling to the $W$ boson and the production with the coupling to the top quark. The analysis of $t H$ production is performed to expose the relative sign of $t-H$ (and $W-H$ ) couplings via this interference. The signal with $H \rightarrow b \bar{b}$ in the final state is defined as a lepton (electron or muon) plus 3 or 4 b-tagged jets and one non-tagged jet. BDT is used to find jet assignment for the $t H q$ signal 

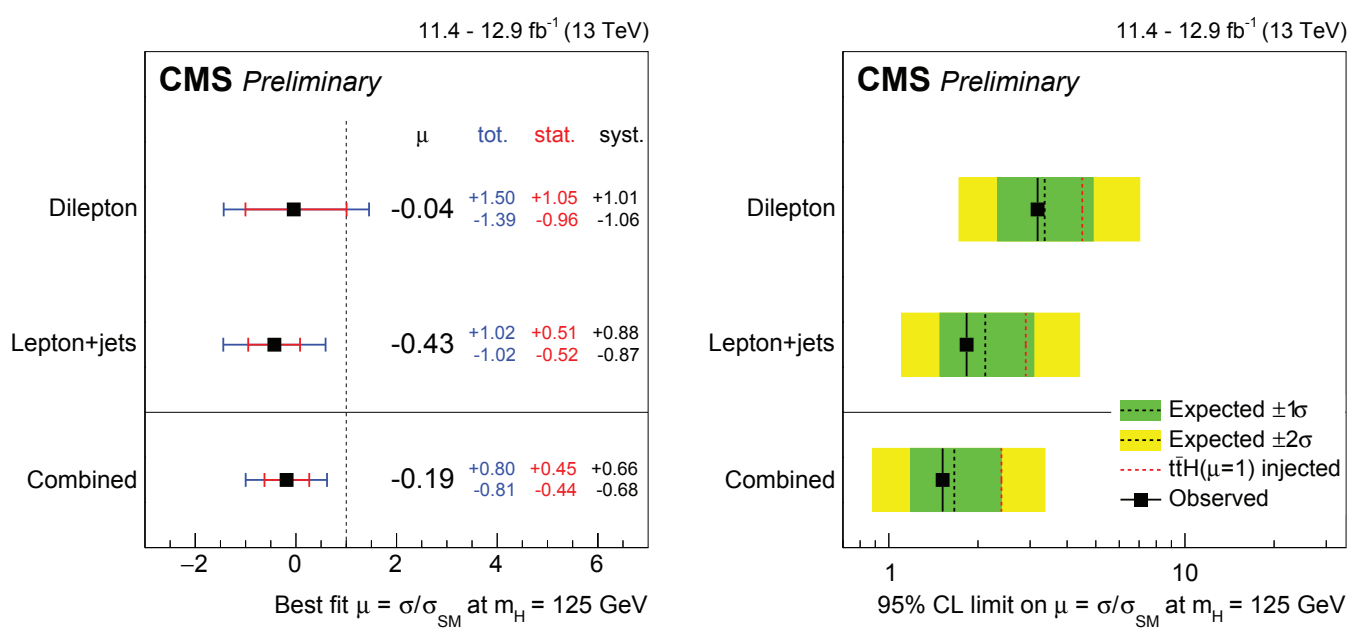

Figure 8. Best-fit values of the signal strength modifiers $\mu$ with their $\pm 1 \sigma$ confidence intervals, also split into their statistical and systematic components (left), and median expected and observed 95\% CL upper limits on $\mu$ (right). The expected limits are displayed together with $\pm 1 \sigma$ and $\pm 2 \sigma$ confidence intervals. Also shown are the limits in case of an injected signal of $\mu=1$ [14].

and the $t \bar{t}$, which represents the main background process. The final results obtained for SM scenario provide 113.7 (98.6) and for the inverted top coupling scenario: 6.0 (6.4), for the observed (and the expected) $95 \% \mathrm{CL}$ upper limit on the cross section for the SM $t H$ production, respectively [17]. In the multilepton final state [18], the strategy is to analyse the events with a single-top quark associated Higgs boson, which decays to $W W, Z Z$ or $\tau \tau$, split into the two categories: two lepton same sign and three lepton. The main irreducible backgrounds are $t \bar{t}+\mathrm{X}$ (where $\mathrm{X}$ can take $W, Z, H$, or $\gamma^{*}$ ), photon conversion, diboson, tri-bosons, and rare SM processes. Reducible backgrounds include fakes from non-prompt leptons and misidentified objects passing lepton selection, as well as the charge flips from charge mis-IDs and the opposite-sign processes (such as $t \bar{t} / Z+$ jets). The $t H q$ process can benefit from a greatly enhanced production cross section in case of anomalous top-Higgs couplings, and the results published in [17] and [18] are used to constrain these couplings.

\subsection{VBF production of the Higgs boson}

$V B F$ production mode with $H \rightarrow b \bar{b}$ is characterized by significantly larger cross section with respect to $V H$ or $t \bar{t} H$, and a very large QCD multijet background. BDT is used for identification of the $V B F$ like events with four jets out of which one or two are b-tagged. The analysis, performed with the $2.3 \mathrm{fb}^{-1}$ of data collected at the energy of $13 \mathrm{TeV}$, provided the signal strength of $\mu=-3.7$, and the other measurement, performed with the $13 \mathrm{TeV}$ data combined with the $8 \mathrm{TeV}$ data of roughly $19 \mathrm{fb}^{-1}$, provided the signal of $\mu=1.3$ [19]. The observed (and expected) upper limits in the absence of a signal are 3.0(5.0) times the SM prediction for the case of the $13 \mathrm{TeV}$ dataset only, and 3.4(2.2) times the SM prediction have been obtained with the combined datasets [19]. 

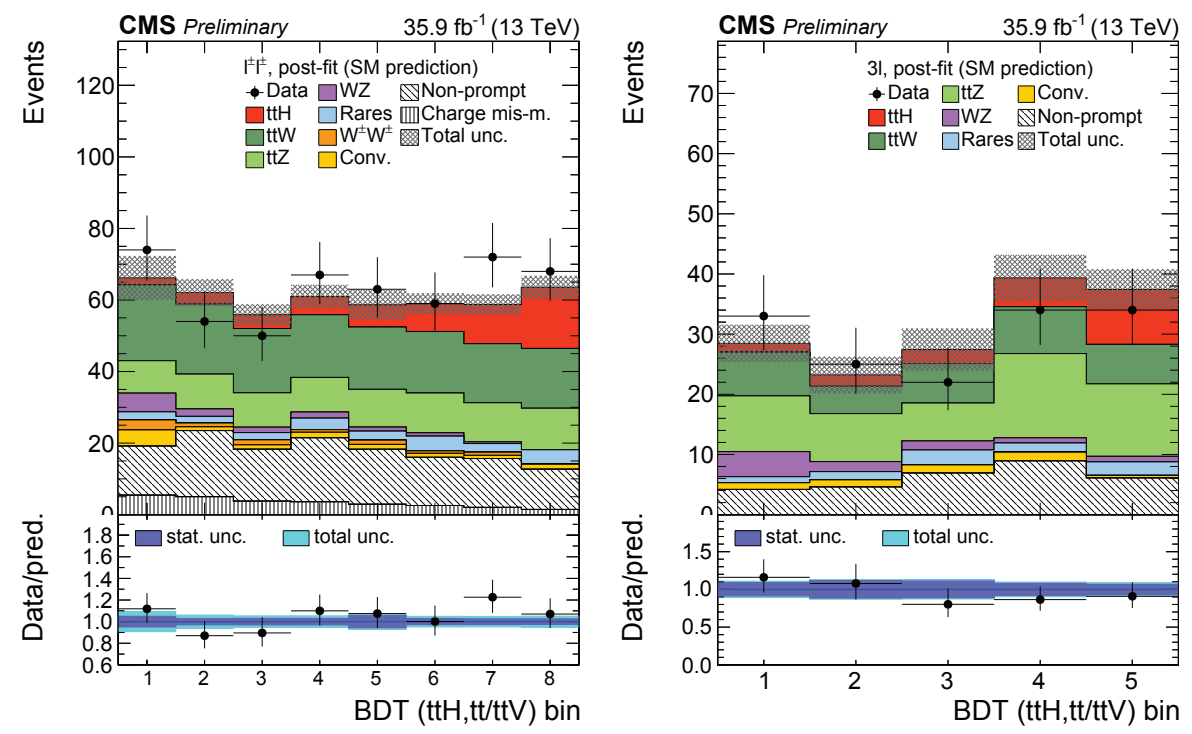

Figure 9. Combination of the BDT classifier outputs in the bins used for signal extraction, for the same-sign di-lepton (left) and three-lepton (right) channels. The distributions are shown after the fit to the data, with all processes constrained to the SM expectation [16].

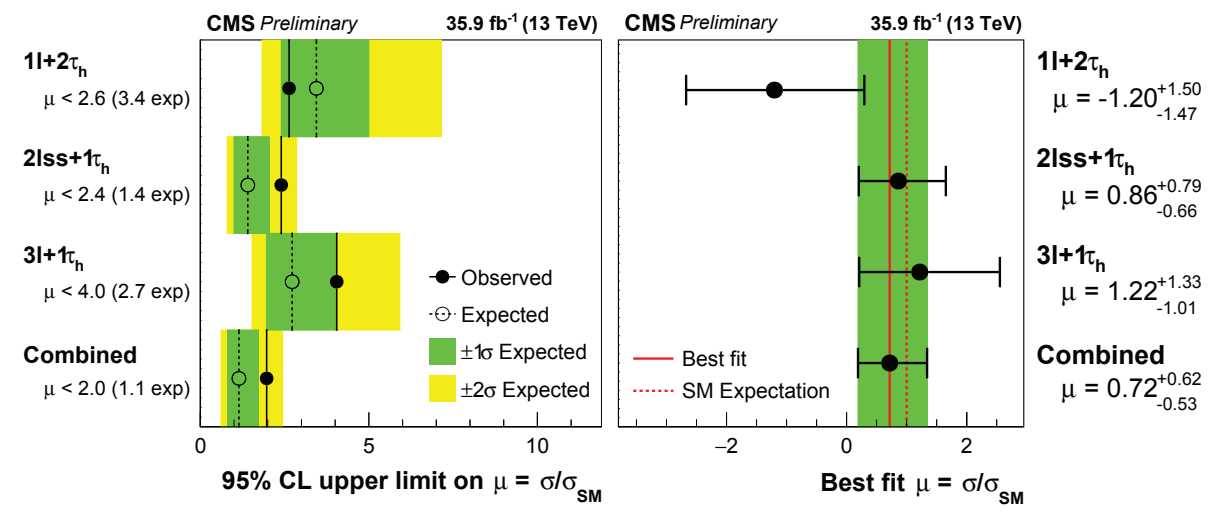

Figure 10. 95\% CL upper limits on the $t \bar{t} H$ signal rate (left), obtained in each of the categories $2 \ell s s+1 \tau_{h}$, $3 \ell+1 \tau_{h}$, and $1 \ell+2 \tau_{h}$ individually and for the combination of all three event categories. The expected limits are computed for the background-only $(\mu=0)$ hypothesis. Signal rates $\mu$ (right), in units of the SM $t \bar{t} H$ production rate, measured in each of the categories $2 \ell s s+1 \tau_{h}, 3 \ell+1 \tau_{h}$, and $1 \ell+2 \tau_{h}$ individually and for the combination of all three categories [15].

\section{Beyond the Standard Model (BSM) Higgs Boson Searches}

Discovery of another scalar boson, neutral or charged, would represent unambiguous evidence for an extended Higgs boson sector. Neutral and charged Higgs bosons appear in many extensions of the SM, of which the simplest are the two Higgs boson doublet models (2HDMs). In a 2HDM, the following five Higgs bosons are predicted: two charged Higgs bosons, $H^{ \pm}$, two neutral scalar (CP-even) Higgs 
bosons, $h$ and $H$, and one neutral pseudoscalar (CP-odd) Higgs boson, $A$. The 2HDM models can be classified into types, depending on the Higgs boson coupling to fermions. In the Type-I 2HDM only one of the Higgs boson doublets couples to fermions, while in a Type-II 2HDM one doublet couples to up-type fermions and the other one to down-type fermions. As a consequence, in Type-I 2HDM the coupling of the charged Higgs bosons to charged leptons and neutrinos is proportional to $\cot \beta$, and in Type-II $2 \mathrm{HDM}$, it is proportional to $\tan \beta$, where $\tan \beta$ represents the ratio of the vacuum expectation values of the two Higgs boson doublets.

The minimal supersymmetric standard model (MSSM), as a special case of a Type-II 2HDM, incorporates a symmetry between bosons and fermions, introducing additional heavier partners for the existing particles, and allowing for possible cancellation of the quadratically divergent self-energy corrections to the Higgs boson mass at high energy, so-called supersymmetry (SUSY). Two free parameters are used to express the MSSM at tree level: the $\tan \beta$ and $m_{A}$ (the mass of the pseudoscalar Higgs boson). Concerning the values of the $\tan \beta$ parameter, while small and medium values represent the models where the gluon fusion is the dominant production mode, a large value means that couplings to down-type fermions are enhanced. This leads to enhanced rate of decays into $\tau$ leptons, and a second dominant production mode, $\mathrm{b}$ associated production. Radiative corrections lead to large modifications of the behaviour beyond tree level, which can depend on a large number of different SUSY parameters. These parameters are fixed to choices that result in different benchmark scenarios, which must produce MSSM models consistent with the discovered $125 \mathrm{GeV}$ Higgs boson. The two scenarios considered in [20] are the $m_{h}^{\bmod +}$ and $h M S S M$. In the case of the $m_{h}^{\text {mod+ }}$ scenario, choices are made for the SUSY parameters which allow the majority of the $m_{A}$-tan $\beta$ plane to be consistent with a $125 \mathrm{GeV}$ scalar Higgs boson. In the case of the $h M S S M$ scenario, the condition of $m_{H}=125 \mathrm{GeV}$ is fixed across the whole plane and the radiative corrections adjusted accordingly.

Another direction would lead to the search for a doubly-charged Higgs boson. The experimental observation of a non-zero neutrino masses [21] contradicts the standard model (SM) assumption of a massless neutrino. There are several models that attempt to expand the SM and accommodate neutrino masses. The minimal Type II seesaw mechanism [22] extends the SM particle spectrum with a scalar triplet with $S U(2)_{L} \times U(1)_{Y}$ quantum numbers $\Phi$. The triplet has a doubly-charged component $\Phi^{ \pm \pm}$, a singly-charged component $\Phi^{ \pm}$, and a neutral component $\Phi^{0}$. The analysis documented in this summary is specific to the left-handed doubly-charged Higgs boson.

A number of models for the physics beyond the SM allow for invisible decay modes of the Higgs boson, such as decays to neutralinos in supersymmetric models [23], graviscalars in models with extra spatial dimensions [24]. More generally, invisible Higgs boson decays can be realized through interactions between the Higgs boson and dark matter (DM) [25]. In Higgs-portal models [26], the Higgs boson acts as a mediator between SM and DM particles allowing for direct production of DM at the LHC. Furthermore, cosmological models proposing that the Higgs boson played a central role in the evolution of the early universe motivate the study of the relationship between the Higgs boson and DM [27].

The BSM physics effects can appear either via anomalous couplings of the Higgs boson or via new particles that can be directly produced or enter in the quantum loop at production. The experimental signature would be an enhancement of the Higgs boson pair (di-Higgs boson) production cross section at a specific invariant mass value (resonant production) or over the whole invariant mass spectrum (non resonant production). The resonant production is predicted by many extensions of the SM such as the Singlet model [28], the Two Higgs boson Doublet Model (2HDM) [29], the minimal supersymmetric standard model (MSSM) [30], and the Warped Extra Dimensions (WED) [31] model. Although the physics motivations and the phenomenology of these theories are very different, the signal is represented as a CP-even scalar particle $(S)$ decaying into a Higgs boson pair, with an intrinsic width that is often negligible with respect to the detector resolution. In the non resonant case, the BSM 
physics is modeled with an effective Lagrangian by extending the SM Lagrangian with dimension-6 operators [32].

A brief overview of the recent results of the BSM Higgs boson searches, essential for the hunt for new physics, follows in the next sections.

\subsection{Search for the charged Higgs boson}

This section summarizes the results of the search for a charged Higgs boson in decays to a $\tau$ lepton and a neutrino based on $12.9 \mathrm{fb}^{-1}$ recorded in 2016 at $\sqrt{s}=13 \mathrm{TeV}$ [33]. The analysis considers the production mode $p p \rightarrow t b H^{ \pm}\left(p p \rightarrow H^{ \pm} W^{\mp} b b\right)$ for masses larger (smaller) than the top quark $\left(m_{H^{ \pm}}>m t-m b\right)$ and focuses on the fully hadronic final state. In this analysis, transverse mass of the Higgs boson has been used for signal extraction. The model independent 95\% CL limits and MSSM $m_{h}^{\text {mod }+}$ exclusion limits in $m_{H^{+}}-\tan \beta$ plane have been presented, where no excess has been observed in the mass range from $80 \mathrm{GeV}-3 \mathrm{TeV}$ and the possibility of the existence of the charge Higgs boson has been excluded in most of the region of interest.

\subsection{MSSM Higgs boson with subsequent decays to a pair of tau leptons}

In the search for the neutral MSSM Higgs boson with the decays into $\tau \tau$ with $12.9 \mathrm{fb}^{-1}$ of data at $\sqrt{s}=13 \mathrm{TeV}$ [20] the following final states: $\tau_{h} \tau_{h}, \mu \tau_{h}, e \tau_{h}$ and $e \mu$, and the two event categories based on the number of b-tagged jets have been considered. Main backgrounds are $Z / \gamma^{*} \tau \tau$, QCD and $t \bar{t}$, and the total transverse mass is used for the signal extraction. The results have been presented in form of the model independent 95\% CL limits and the exclusion limits in the $m_{A}-\tan \beta$ plane, in the context of the $m_{h}^{\text {modt }}$ and $h M S S M$ scenario. No excess has been found in the mass range $100 \mathrm{GeV}-3 \mathrm{TeV}$, and with the complete 2016 dataset, a larger part of the $m_{A}-\tan \beta$ plane has been excluded.

\subsection{Doubly-charged Higgs boson searches}

A search for doubly-charged Higgs boson production in three and four lepton final states at $\sqrt{s}=$ $13 \mathrm{TeV}$ is performed for a narrow di-lepton resonance in pair production and associated production modes: $q \bar{q} \rightarrow Z^{0} / \gamma \rightarrow \Phi^{++} \Phi^{--} \rightarrow \ell_{i}^{+} \ell_{j}^{+} \ell_{k}^{-} \ell^{-} l$, and $q q^{\prime} \rightarrow W^{ \pm} \rightarrow \Phi^{ \pm \pm} \Phi^{\mp} \rightarrow \ell_{i}^{ \pm} \ell_{j}^{ \pm} \ell_{k}^{\mp} v_{l}$. Measuring the branching fractions of the doubly-charged Higgs boson would give access to neutrino parameters such as the masses $\left(m_{1}, m_{2}, m_{3}\right)$, the Majorana Phases $\left(\alpha_{1}, \alpha_{2}\right.$, or $\left.\Delta \alpha=\left|\alpha_{1}-\alpha_{2}\right|\right)$, the CP-violating angle $\delta$, and the Higgs boson triplet vacuum expectation value. Since the branching fractions are not fixed by the model, model independent limits are set assuming $100 \% \Phi^{++}$decays to a single pair of leptons: $e e, e \mu, \mu \mu, e \tau, \mu \tau$, and $\tau \tau$. Limits have been also set for four benchmark points that target different neutrino mass hierarchies. As the final result of this search, no significant excess has been observed. Lower bounds on the $\Phi^{ \pm \pm}$mass are extended to between $800 \mathrm{GeV}$ and $820 \mathrm{GeV}$ for final states with $100 \%$ decays to $e e, e \mu$, and $\mu \mu$, and to $714 \mathrm{GeV}$ and $643 \mathrm{GeV}$ for final states with $100 \%$ decays to e $\tau$ and $\mu \tau$, respectively. Final states with $100 \%$ decays to $\tau \tau$ are excluded up to $535 \mathrm{GeV}$. In addition, benchmark points of the type II seesaw model targeting four possible neutrino mass hypotheses are also probed, with lower bounds between $716 \mathrm{GeV}$ and $761 \mathrm{GeV}$ [34].

\subsection{Invisible Higgs boson}

Analyses of the invisible Higgs boson decays target different Higgs boson production modes: $V B F$ production with two forward jets, $V H$ production with $Z \rightarrow e e / \mu \mu$ [35], $Z \rightarrow b \bar{b}, W / Z \rightarrow q \bar{q}$, or gluon 
Table 1. Comparison table of the $13 \mathrm{TeV}$ non-resonant observed (expected) $\sigma / \sigma_{S M} 95 \%$ CL limits for $b \bar{b} \tau \tau$ [37], $b \bar{b} \ell v \ell v$ [38], $b \bar{b} \gamma \gamma$ [39] and $b \bar{b} b \bar{b}$ [40] channels.

\begin{tabular}{lcc}
\hline channel & limit & integrated luminosity \\
\hline$b \bar{b} \tau \tau$ & $28(25)$ & $35.9 \mathrm{fb}^{-1}$ \\
$b \bar{b} \ell \nu \ell v$ & $79(89)$ & $35.9 \mathrm{fb}^{-1}$ \\
$b \bar{b} \gamma \gamma$ & $91(90)$ & $2.7 \mathrm{fb}^{-1}$ \\
$b \bar{b} b \bar{b}$ & $342(308)$ & $2.3-2.7 \mathrm{fb}^{-1}$ \\
\hline
\end{tabular}

fusion in association with the initial state radiation have been presented. The observed/expected upper limits at $95 \% \mathrm{CL}$ on $B(H \rightarrow$ invisible $)$ of $0.24 / 0.23$ for the combination of 7,8 and $13 \mathrm{TeV}$ with the 2015 dataset measurements, then $0.40 / 0.42$ for the $Z(l l)+M E T$ analysis and $0.53 / 0.40$ for the monojet and $V(j j)$ analyses updated with full 2016 dataset [36] have been shown in this report.

\subsection{Di-Higgs boson}

In CMS, both di-Higgs boson productions, resonant and non-resonant, have been investigated. Many $\mathrm{BSM}$ models predict resonant $\mathrm{X} \rightarrow H H$ production, while non-resonant SM di-Higgs boson production provides a crucial test of electroweak symmetry breaking. The results of the following five searches for the Higgs boson pair production in proton-proton collisions at $\sqrt{s}=13 \mathrm{TeV}$ have been reported. The search for pair production of Higgs bosons in the two $\tau$ leptons and two bottom quarks final state [37], the search for resonant and non-resonant Higgs boson pair production in the $b \bar{b} \ell v \ell v$ final state [38] and the search for $H(b \bar{b}) H(\gamma \gamma)$ decays [39], besides a pair of leptons or photons in the final state, require a decay of $H \rightarrow b \bar{b}$ to keep branching fraction higher. The highest branching fraction is obtained in the search for resonant pair production of Higgs bosons decaying to two bottom quark-antiquark pairs [41] and the search for heavy resonances decaying to a pair of Higgs bosons in the four b quark final state [42], where the first one targets low mass resonances between 260 and $1200 \mathrm{GeV}$, and the other one targets the masses between 800 and $3000 \mathrm{GeV}$.

The current status of the results on $13 \mathrm{TeV}$ non-resonant observed (expected) $\sigma / \sigma_{S M} 95 \% \mathrm{CL}$ limits (where [37] and [38] have been updated with the full 2016 datasets) is shown in the Tab. 1. A comparison between the summary of the Run-II $\sigma(g g \rightarrow X) \times B(X \rightarrow H H) 95 \%$ CL upper limits from the five resonant di-Higgs boson searches [43] and the results of the corresponding Run I analyses has been presented in this report.

\section{Higgs Boson Searches Prospects}

This section is related to the updates on projections of physics reach with the upgraded CMS detector for HL-LHC [44]. Selected measurements in Higgs boson physics, highlighting the performance of the planned upgrades of the CMS detector, have been presented. Public results are extrapolated to larger data sets 300 and $3000 \mathrm{fb}^{-1}$. In order to summarize the future physics potential of the CMS detector at the HL-LHC, extrapolations have been made under different uncertainty scenarios: $\mathrm{S} 1, \mathrm{~S} 1+, \mathrm{S} 2$ and $\mathrm{S} 2+$, where S1 denotes the scenario with all systematic uncertainties kept constant with integrated luminosity, and $\mathrm{S} 2$ denotes theoretical uncertainties scaled down by a factor $1 / 2$ and experimental systematic uncertainties scaled down by the square root of the integrated luminosity (until they reach a defined lower limit based on estimates of the achievable accuracy with the upgraded detector). The "+" denotes taking into account the effects of higher pileup conditions and detector upgrades on the future CMS performance, instead of leaving the performance of the CMS detector 
unchanged with respect to the reference analysis. In the following text the future prospects for the SM Higgs boson production in the $H \rightarrow \gamma \gamma$ and $H \rightarrow Z Z \rightarrow 4 \ell$ analysis channels have been described.

According to the measurements of Higgs boson properties using the $H \rightarrow \gamma \gamma$ decay, with $12.9 \mathrm{fb}^{-1}$ of data collected in 2016 [45], the following projections from the analysis have been made: the signal strength (inclusively and per production mode, presented in this report), Higgs boson coupling strength modifiers and the fiducial cross section measurement [44]. Projected symmetrized uncertainties for the $H \rightarrow \gamma \gamma$ signal strength relative to the standard model $\left(\mu_{\gamma \gamma}\right)$, inclusively and per production mode (VBF, $t \bar{t} H$ and $g g H$ ) are shown. No projections for the VH signal strength is given because the reference analysis [45] was not sensitive to it. For the $3000 \mathrm{fb}^{-1}$ case the effect of the high pileup conditions of the HL-LHC has been taken into account as degradations to the photon identification efficiency and vertex identification efficiency. In each case the uncertainty for the inclusive signal strength is shown split into statistical uncertainties, experimental systematic uncertainties and theoretical systematic uncertainties component. In S2 and S2+, the experimental uncertainty on the integrated luminosity is reduced to $1.5 \%$.

According to the measurements of Higgs boson properties using $H \rightarrow Z Z \rightarrow 4 \ell$ with $12.9 \mathrm{fb}^{-1}$ of data collected in 2016 [46], the following projections from the analysis have been made: the signal strength per production mode (presented in this report), differential cross section measurement for $p_{T}(H)$ and the constraints on anomalous $H \rightarrow Z Z$ couplings [44]. The projected $68 \%$ CL uncertainties on the Higgs boson signal strength for different production modes at 300 and $3000 \mathrm{fb}^{-1}$, with S1 and $\mathrm{S} 2$ have been presented. In S2, the experimental uncertainties on the integrated luminosity and the lepton identification efficiency are reduced to $1.5 \%$ and $1 \%$ per lepton, respectively. The projections for $3000 \mathrm{fb}^{-1}$ use different lepton efficiencies and misidentification rates to account for the higher pileup at the HL-LHC.

The uncertainties are in general dominated by statistical component, resulting in 3-4 times lower total uncertainties with respect to the ones from the Run I measurements for $300 \mathrm{fb}^{-1}$, and 5-10 times lower for the high luminosity measurements, with $3000 \mathrm{fb}^{-1}$. Further information on the projections made for top physics, heavy flavor physics, searches for dark matter and new heavy particles can be found in the CMS Detector Performance Summary [44].

\section{Summary}

As a conclusion from the presented results of the SM Higgs boson searches it follows that the precision of the $H \rightarrow \gamma \gamma$ and $H \rightarrow Z Z \rightarrow 4 \ell$ analyses has been improved and the SM process $H \rightarrow \tau \tau$ has been observed. The sensitivity of the search for the production of the Higgs boson associated to top quarks has been increased and no significant deviations from the SM, neither extra Higgs bosons, nor a sign of the BSM physics have been yet found. As for the outlook, the updates of several important analyses with the full 2016 dataset are expected, looking forward for around $100 \mathrm{fb}^{-1}$ of data to be collected by the end of the LHC Run II.

\section{References}

[1] CMS Collaboration, JINST 3 S08004 (2008)

[2] CMS Collaboration, Eur. Phys. J. C75, 5, 212 (2015)

[3] CMS and ATLAS Collaboration, JHEP, 08, 045 (2016)

[4] CMS Collaboration, CMS-PAS-HIG-16-040 (2017), http://cds.cern.ch/record/2264515

[5] CMS Collaboration, CMS-PAS-HIG-17-015 (2017), http://cds.cern.ch/record/2257530

[6] LHC Higgs Cross Section Working Group, arXiv:1610.07922 (2016) 
[7] CMS Collaboration, CMS-PAS-HIG-16-041 (2017), http://cds.cern.ch/record/2272260

[8] CMS Collaboration, JHEP 1604 (2016) 005

[9] Alioli, Simone and Nason, Paolo and Oleari, Carlo and Re, Emanuele, JHEP, 07, 060 (2008)

[10] Nason, Paolo, JHEP, 11, 040 (2004)

[11] Frixione, Stefano and Nason, Paolo and Oleari, Carlo, JHEP, 11, 070 (2007)

[12] ATLAS and CMS Collaborations, Phys.Rev.Lett. 114 (2015) 191803

[13] CMS Collaboration, CMS-PAS-HIG-16-043 (2017), http://cds.cern.ch/record/2276465

[14] CMS Collaboration, CMS-PAS-HIG-16-038 (2016), http://cds.cern.ch/record/2231510

[15] CMS Collaboration, CMS-PAS-HIG-17-003 (2017), http://cds.cern.ch/record/2257067

[16] CMS Collaboration, CMS-PAS-HIG-17-004 (2017), http://cds.cern.ch/record/2256103

[17] CMS Collaboration, CMS-PAS-HIG-16-019 (2016), http://cds.cern.ch/record/2204925

[18] CMS Collaboration, CMS-PAS-HIG-17-005 (2017), http://cds.cern.ch/record/2264553

[19] CMS Collaboration, CMS-PAS-HIG-16-003 (2016), http://cds.cern.ch/record/2160154

[20] CMS Collaboration, CMS-PAS-HIG-16-037 (2016), http://cds.cern.ch/record/2231507

[21] Olive, K. A. and others, Chin. Phys., C38, 090001 (2014)

[22] Raidal, M. and others, Eur. Phys. J., C57, 13-182 (2008)

[23] Belanger, G. and Boudjema, F. and Cottrant, A. and Godbole, R. M. and Semenov, A., Phys. Lett., B519, 93-102 (2001)

[24] Giudice, Gian F. and Rattazzi, Riccardo and Wells, James D., Nucl. Phys., B595, 250-276 (2001)

[25] Shrock, Robert E. and Suzuki, Mahiko, Phys. Lett., 110B, 250 (1982)

[26] Baek, Seungwon and Ko, P. and Park, Wan-Il and Senaha, Eibun, JHEP, 05, 036 (2013)

[27] Servant, Geraldine and Tulin, Sean, Phys. Rev. Lett., 111, 151601 (2013)

[28] Binoth, T. and van der Bij, J. J., Z. Phys., C75, 17-25 (1997)

[29] Branco, G. C. and Ferreira, P. M. and Lavoura, L. and Rebelo, M. N. and Sher, Marc and Silva, Joao P., Phys. Rept., 516, 1-102 (2012)

[30] Fayet, Pierre, B90, 104-124 (1975)

[31] Agashe, Kaustubh and Davoudiasl, Hooman and Perez, Gilad and Soni, Amarjit, Phys. Rev., D76, 036006 (2007)

[32] Goertz, Florian and Papaefstathiou, Andreas and Yang, Li Lin and Zurita, Jose, JHEP, 04, 167 (2015)

[33] CMS Collaboration, CMS-PAS-HIG-16-031 (2016), http://cds.cern.ch/record/2223865

[34] CMS Collaboration, CMS-PAS-HIG-16-036 (2017), http://cds.cern.ch/record/2242956

[35] CMS Collaboration, CMS-PAS-EXO-16-052 (2017), http://cds.cern.ch/record/2264690

[36] CMS Collaboration, JHEP, 02, 135 (2017)

[37] CMS Collaboration, CMS-PAS-HIG-17-002 (2017), http://cds.cern.ch/record/2273798

[38] CMS Collaboration, CMS-PAS-HIG-17-006 (2017), http://cds.cern.ch/record/2278570

[39] CMS Collaboration, CMS-PAS-HIG-16-032 (2016), http://cds.cern.ch/record/2207960

[40] CMS Collaboration, CMS-PAS-HIG-16-026 (2017), http://cds.cern.ch/record/2209572

[41] CMS Collaboration, CMS-PAS-HIG-16-002 (2016), http://cds.cern.ch/record/2141024

[42] CMS Collaboration, CMS-PAS-B2G-16-026 (2017), http://cds.cern.ch/record/2264684

[43] Higgs PAG Summary Plots, http://twiki.cern.ch/twiki/bin/view/CMSPublic/SummaryResultsHIG

[44] CMS Collaboration, CMS-DP-2016-064 (2016), http://cds.cern.ch/record/2221747

[45] CMS Collaboration, CMS-PAS-HIG-16-020 (2016), http://cds.cern.ch/record/2205275

[46] CMS Collaboration, CMS-PAS-HIG-16-033 (2016), http://cds.cern.ch/record/2204926 